\title{
Numerical Simulation of Cavitation Inception on Radial Flow Pump
}

\author{
D. Somashekar ${ }^{1}$, Dr. H. R. Purushothama ${ }^{2}$ \\ ${ }^{I}$ (M.Tech Student, Siddaganga Institute of Technology (SIT), Tumkur, Karnataka 572103. India) \\ 2 (Associate Professor, Department of Mechanical Engineering, Siddaganga Institute of Technology (SIT),
} Tumkur, Karnataka 572103).

\begin{abstract}
Pump cavitation is the formation and subsequent collapse or implosion of vapor bubbles in a pump. It occurs when the absolute pressure on the liquid falls below the liquid's vapor pressure. This paper describes the validation of cavitation inception on a radial flow pump which develops $21 \mathrm{~m}$ of head at 26lps of discharge at its best efficiency point (BEP) of discharge while operating at a speed of 1500rpm. Design, operation and refurbishment of centrifugal pumps are strongly related to cavitation flow phenomena, which may occur in either the rotating runner-impeller or the stationary parts of the centrifugal pumps. The numerical simulation (ANSYS CFX software Release 13) is used to study the cavitation inception in centrifugal pump by varying water level in the cavitation well at constant speed condition. The computational grid was generated by $C F X-$ Turbo grid with $\mathrm{H}$-Grid through the blade and flow passage. The flow simulation is executed in the rotation frame of reference. Standard $(k-\varepsilon)$ model is used for the turbulence. Turbulent wall treatment; the volume of fluid model is selected for cavitation model. Upwind difference is used for solution scheme. Numerical results will be compared with experimental data in terms of head and NPSH.
\end{abstract}

Keywords: ANSYS CFX software Release 13, CFD, Inception, NPSH, Radial Flow Pump, Turbulence Model ( $k$ ह)

\section{Introduction}

Larry Bachus., and Angel Custodio [15] when the vapor bubbles collapse with high enough frequency, it sounds like marbles and rocks are moving through the pump. If the vapor bubbles collapse with high enough energy, they can remove metal from the internal casing wall, and leave indent marks appearing like blows from a large ball pein hammer. Inadequate NPSH available (NPSHa) is a root cause for cavitation in the pump. Insufficient NPSH leads to lower liquid pressure in the pump and if pressure in the eye of the impeller falls below the vapor pressure of the fluid, then cavitation can begin by formation of vapor bubbles

S Christopher and S Kumaraswamy [1] experimentally studied the Cavitation Hysteresis on Radial Flow Pump for varying head and discharge. In this paper the experimental results provided a clear understanding of the onset/cessation and development/reduction of cavitation while the water level in sump was decreased/ increased. The difference for these two operation is termed as "cavitation Hysteresis" in a radial flow pump. Tulin [2, 3] first introduced a linear theory to analyze the cavitating flow around a two-dimensional hydrofoil. This theory somewhat resembles the thin wing theory. It assumes that the cavity and the foil thicknesses are thin, compared to the foil chord length. Based on this assumption, the dynamic boundary condition on the cavity surface can be significantly simplified by specifying a constant horizontal perturbation velocity there. Geurst [4] employed the conformal transformation technique to derive theoretical expressions of the lift, drag, and moment coefficients, each of which contained coefficients in integral form. He also studied the special case of a flat-plate hydrofoil and obtained simple analytical expressions of these coefficients in terms of the angle of attack of inflow and the transformation parameters. Nevertheless, all these results apply only to the flow at a small angle of attack, as the assumptions of the linear theory imply. With the modern evolution of computational methods, several nonlinear numerical procedures of boundary-element type have been successfully developed for the solution of sheet cavitation, based on the early theoretical achievements. In these approaches, cavity surface conditions are usually satisfied on the exact cavity surface that is part of the solution and determined iteratively by proper computational algorithms. Uhlman $[\mathbf{5 , 6} \mathbf{6}$ employed a velocity-based nonlinear boundary element method to obtained solutions for partially-cavitating and supercavitating hydrofoil flows. Kinnas and Fine [7] developed a potential-based nonlinear boundary element method developed for the non-linear analysis of inviscid cavitating flow around hydrofoils or propeller blades. GuoyuWang,et.al[8] experimentally studied stationary and non-stationary characteristics of attached, turbulent cavitating flows around solid objects. Different cavitation regimes, including incipient cavitation with traveling bubbles, sheet cavitation, cloud cavitation, and supercavitation, are addressed along with both visualization and quantitative information. Phenomena such as large-scale vortex structure and rear re-entrant jet associated with cloud 
cavitation, and subsequent development in supercavitation are described. Khin Cho Thin et.al.[9] have carried out computational analysis of a centrifugal pump and predicted performance for off-design volume flow rate and calculated impeller volute disc friction loss, slip, shock losses, recirculation losses and other friction losses. Mohammed Khudhair Abbas[10] employed the computational grid generated by SFX Turbogrid with H-Grid through the blade and flow in passage of centrifugal pump and cavitation model. Shen and Dimotakis 's work[11] have been widely used to evaluate commercial codes. Their data show the pressure distribution over hydrofoil in the GALCIT cavitation tunnel. There is only one pressure tap over pressure side in their hydrofoil, so the information of the flow around the pressure surface is limited. S.Rajendran and Dr.K.Purushothaman[12] have carried out the analysis of centrifugal pump impeller design is carried out using ANSYS-CFX. The complex internal flows in Centrifugal pump impellers can be well predicted through ANSYS-CFX. The numerical solution of the discredited three-dimensional, incompressible Navier- Stokes equations over an unstructured grid is accomplished with an ANSYS-CFX. The flow pattern, pressure distribution in the blade passage, blade loading and pressure plots are discussed in this paper. Modeling guide[13] the k-epsilon Model

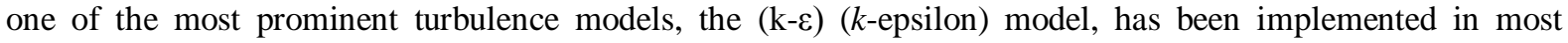
general purpose CFD codes and is considered the industry standard model. It has proven to be stable and numerically robust and has a well-established regime of predictive capability. For general purpose simulations, the model offers a good compromise in terms of accuracy and robustness. Within CFX, the (k- $\varepsilon$ ) turbulence model uses the scalable wall-function approach to improve robustness and accuracy when the near-wall mesh is very fine. The scalable wall functions allow solution on arbitrarily fine near wall grids, which is a significant improvement over standard wall functions. Bruno Schiavello and Frank[14] Pump cavitation, discussing various net positive suction head required (NPSHR) criteria, net positive suction head available (NPSHA) margins and impeller life expectancy. It gives an introduction to the subject matter and provides insights on particulars like cavitation inception, 3 percent head drop, and 40,000 hours impeller life, as well as NPSH scaling laws. It further devotes attention to the effect of dissolved gases and thermal suppression (i.e., thermodynamic effect). With regard to numerical prediction capabilities the use of computational fluid dynamics (CFD) shall be discussed. Incipient cavitation (NPSHi) [14] The incipient cavitation characteristic plays a key role when designing and evaluating centrifugal impellers with regard to suction performance, pumps can-and will-operate satisfactorily for a certain period of time when NPSHR < NPSHA < NPSHi. However, there may be applications where it is desired to have NPSHi < NPSHA over a particular flow range and thus ensure a cavitation free region of operation. The objective of the pump designer will then be to establish a design with its best cavitation point somewhat beyond the design operating capacity, Qdesign, and have NPSHi < NPSHA. Depending on the desired run-out capacity and the volumetric efficiency of the pump/impeller combination the ratio QBEP/Qdesign can be upto 120 to 125 percent, or even further. A high QBEP/Qdesign ratio, however, places a serious constraint on the NPSHi that can be met at design capacity, and may even make it impossible to achieve NPSHi < NPSHA at Qdesign. To determine the incipient cavitation characteristic there are several options, including: a.Flow visualization, b. Hydro-acoustic noise measurement, c. Numerical flow simulations.

\section{Factors Effect the cavitation inception [Ref-1]}

There are many complex factors influencing inception and development of cavitation are as follows,

1. Effect of air content: It is possible that the undissolved air present in the liquid may be playing an important role in this case.2.Effect of dimensionless-tension parameter, 3.Effect of velocity, 4.Effect of surface characteristics and 5.Effect of history.

\section{Geometric Modeling of Pump}

In order to obtain better design in CFD, following procedure is applied so that fluid flow can easily be modelled. Initial design of the model is a planning decision and the geometry is generated depending on these initial design considerations, using either CFD modelling tools or other Design tools. The first task to accomplish in a numerical flow simulation is the definition of the geometry, followed by the grid generation.

Table.1 Centrifugal pump design parameters [Ref-1]

\begin{tabular}{|l|l|}
\hline Type & Open \\
\hline Inlet Diameter & $147 \mathrm{~mm}$ \\
\hline Outlet Diameter & $307 \mathrm{~mm}$ \\
\hline Inlet Vane angle & 23 Degree \\
\hline Outlet Vane angle & 30 Degree \\
\hline Inlet Width & $18.5 \mathrm{~mm}$ \\
\hline Outlet Width & $8.5 \mathrm{~mm}$ \\
\hline Blade thickness & $5 \mathrm{~mm}$ \\
\hline Number of blades & 6 \\
\hline Speed & $1500 \mathrm{rpm}$ \\
\hline
\end{tabular}


This step is the most important step for the study of an isolated impeller assuming an axis symmetric flow simplifies the domain to a single blade passage. The geometry of design needs to be created from the initial design. Any modeling software can be used for modeling and then shifted to other simulation software for analysis purposes. Before the modeling of blade, a generalized program is written for the design of the blade. The flow simulation is executed in the rotation frame of reference. Standard $(\mathrm{k}-\varepsilon)$ model is used for the turbulence. The parameters which were considered initially are Head, Flow Rate, Pump Speed, Volumetric efficiency and Overall Efficiency. The Centrifugal pump design parameters are given in the Table1. Some of the output parameters of this design were used as input of the Blade Modeller. Fig.1. and Fig.2 shows the Geometric Model pump with volute casing.

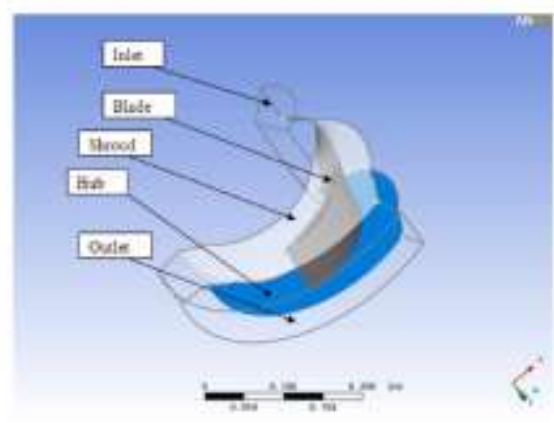

Fig. 1 Geometric model of pump impeller

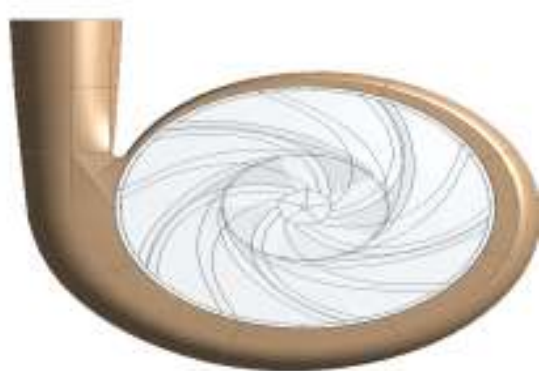

Fig. 2 Geometric model pump with volute casing

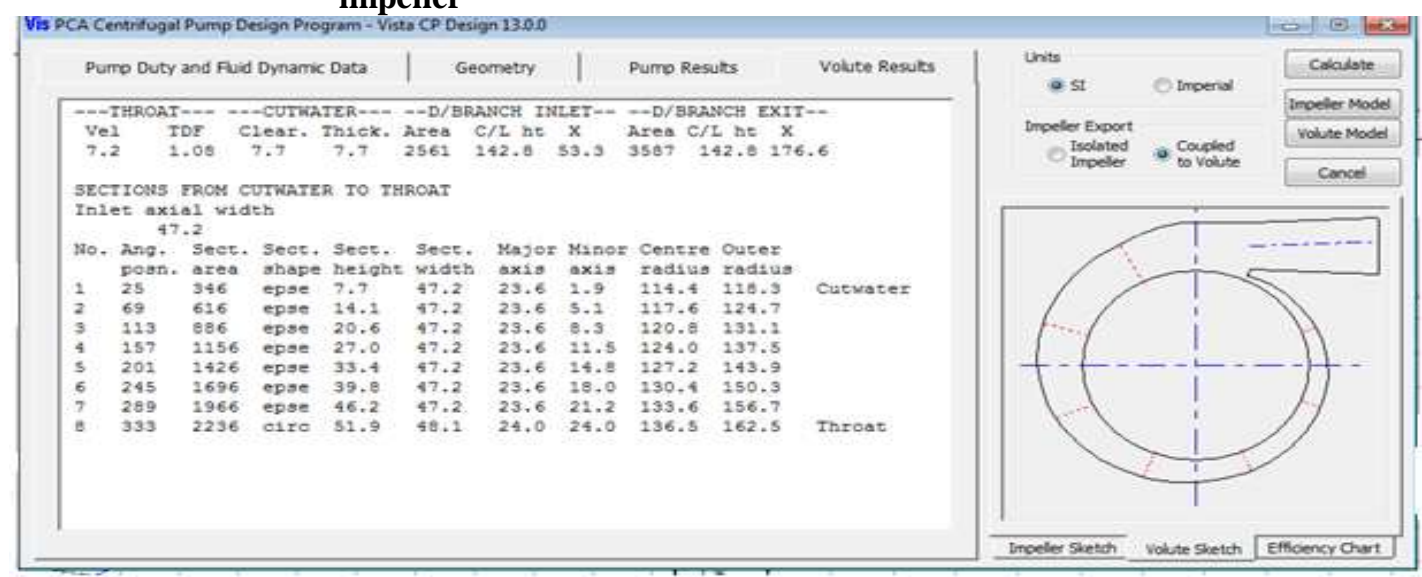

Fig.3 Blade modeller diffuser design model

Mesh generation (Girding) is the process of subdividing a region to be modelled into a setoff small control volumes. Associated with each control volume there will be one or more values of the dependent flow variables (e.g., velocity, pressure, temperature, etc.) Usually these represent some type of locally averaged values. Numerical algorithms representing approximations to the conservation laws of mass, momentum and energy are then used to compute these variables in each control volume.

Meshing is a method to define and break up the model into small elements. In general, a finite element model is defined by a mesh network, which is made up of the geometric arrangement of elements and nodes. Nodes represent points at which features such as displacements are calculated. Elements are bounded by sets of nodes, and define localized mass and stiffness properties of the model.

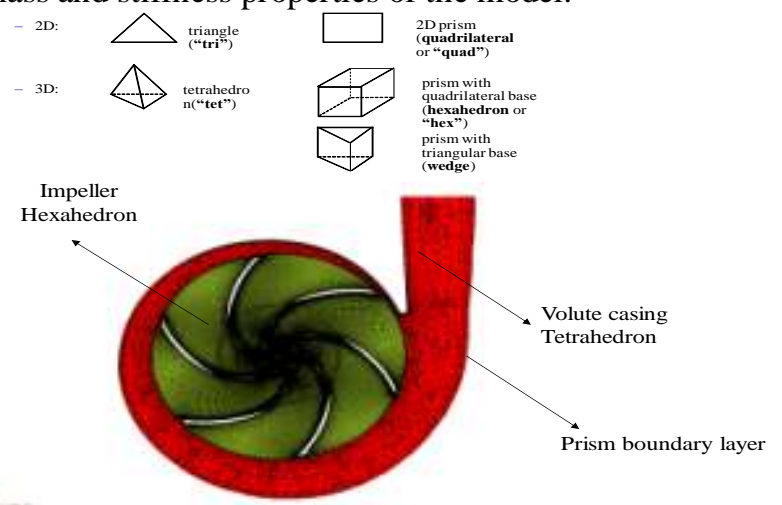

Fig. 4 Meshing details of centrifugal pump -sectional view 
Elements are also defined by mesh numbers, which allow references to be made to corresponding deflections, stresses, pressures, temperatures at specific model locations. The traditional method of mesh generation is block-structure (multi-block) mesh generation. The block-structure approach is simple and efficient technique of mesh generation. Fig. 4 shows the Meshing details of Centrifugal pump -Sectional view.

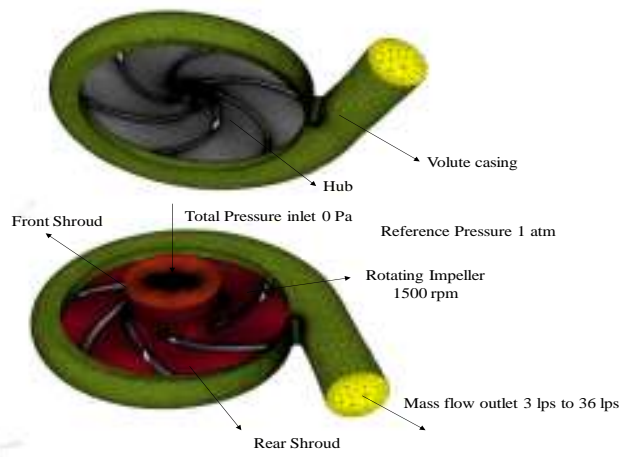

Fig. 5 Boundary conditions centrifugal pump

\section{Results and Discussions}

The pressure increases gradually along stream wise direction within impeller passage and has higher pressure in pressure side than suction side of the impeller blade. However, the pressure developed inside the impeller is not so uniform. The isobar lines are not all perpendicular to the pressure side of the blade inside the impeller passage; this indicated that there could be a flow separation because of the pressure gradient effect. The fig.6 shows the pressure distribution within the impeller and pump.
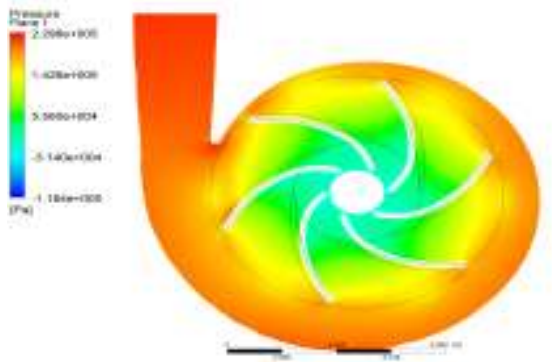

Fig. 6 Pressure distribution middle plane of pump

Velocity also increases gradually along streamwise direction within the impeller passage. As the flow enters the impeller eye, it is diverted to the blade-to-blade passage. The flow at the entrance is not shockless because of the unsteady flow entering the impeller passage. The separation of flow can be seen at the blade leading edge. Since, the flow at the inlet of impeller is not tangential to the blade, the flow along the blade is not uniform and hence the separation of flow takes place along the surface of blade. Here it can be seen that flow separation is taking place on both side of the blade, ie, pressure and suction side as shown in Fig.7.

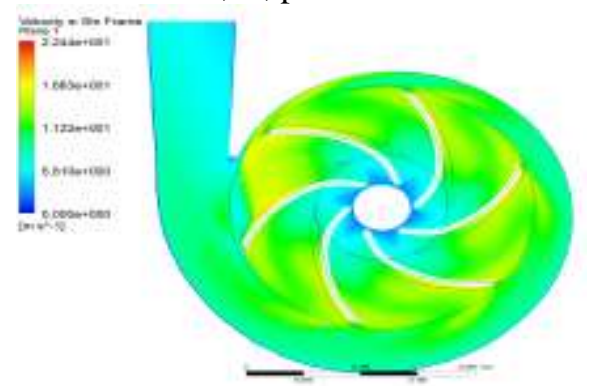

Fig. 7 Velocity distribution at pump

The Fig. 8 shows the head-flow curve for water as fluid in the impeller flows at different flow rates. It is found that the head of pump decreases gradually as the flow rate in the pump increases Fig. 9 shows that the stages of water- vapor fringe plot 3D view at $99 \%$ span that the cavitation is form ,initially no cavitation appear then develop to reach maximum when static pressure reduce below vapor pressure.

To clarify cavitation phenomenon in flow passage in centrifugal pump, the compute static pressure is plotted in blade to blade view at NPSH value of $(7 \mathrm{~m})$ that inception cavitation occurred at the blade leading edge near the tip, figure 10 shows that the pressure fringe plot $3 \mathrm{D}$ view in this region the static pressure reduce below vapor pressure when the flow field static pressure decrease below liquid vapor pressure, cavitation will be 
formation. In this figure, the results of numerical simulation are shown along with the experimental data from Ref.1.

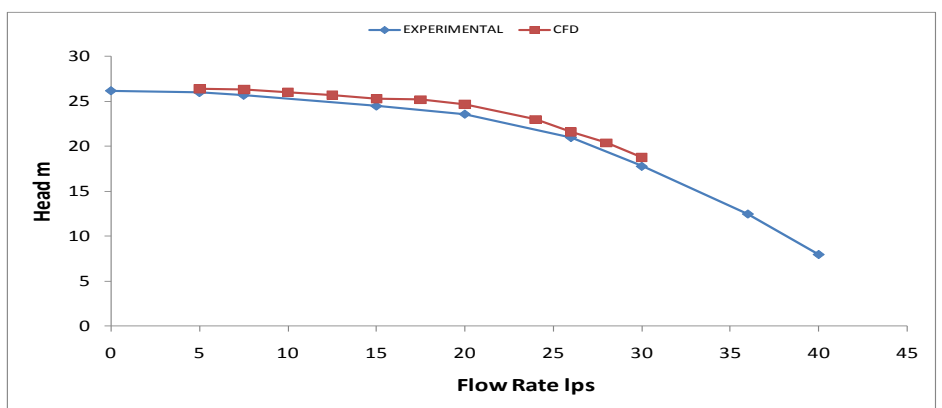

Fig. 8 Head v/s flow rate without cavitation [Experimental value Ref-1]

To avoid cavitation in centrifugal pumps, the pressure of the fluid at all points within the pump must remain above saturation pressure. The quantity used to determine if the pressure of the liquid being pumped is adequate to avoid cavitation is the net positive suction head (NPSH). The net positive suction head available (NPSHA) is the difference between the pressure at the suction of the pump and the saturation pressure for the liquid being pumped. The net positive suction head required (NPSHR) is the minimum net positive suction head necessary to avoid cavitation

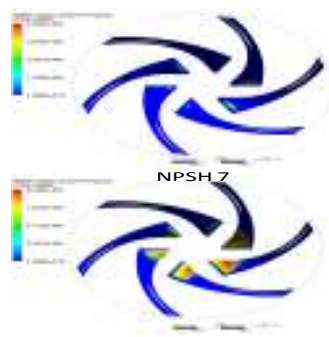

NPSH 3

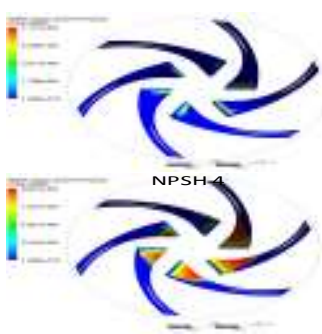

NPSH 2.5

Fig. 9 Comparison of vapour contours of cavitation at $26 \mathrm{lps}, 1500 \mathrm{rpm}$

The condition that must exist to avoid cavitation is that the net positive suction head available must be greater than or equal to the net positive suction head required.

A formula for NPSHA can be stated as the following equation. NPSHA = Psuction - Psaturation .Eq.1[Ref-14]

When a centrifugal pump is taking suction from a tank or other reservoir, the pressure at the suction of the pump is the sum of the absolute pressure at the surface of the liquid in the tank plus the pressure due to the elevation difference between the surface of liquid in the tank and the pump suction less the head losses due to friction in the suction line from the tank to the pump. NPSHA $=$ Pa + Pst - hf - Psat Eq.2 [Ref-14]

Where: NPSHA $=$ Net positive suction head available, $\mathrm{Pa}=$ Absolute pressure on the surface of the liquid Pst $=$ Pressure due to elevation between liquid surface and pump suction, $\mathrm{hf}=$ Head losses in the pump suction piping , Psat $=$ Saturation pressure of the liquid being pumped

It can be use numerical simulation (ANSYS CFX software) to detect the cavitation in centrifugal pump. Figure 11 shows head drop curve as well as curve that computed by ANSYS CFX at design flow and rate speed. Volume of fluid model is selected as a cavitation model to compute rate of vapor bubble production. At inlet boundary total pressure is decreased to meet a status that cavitation formation starting.
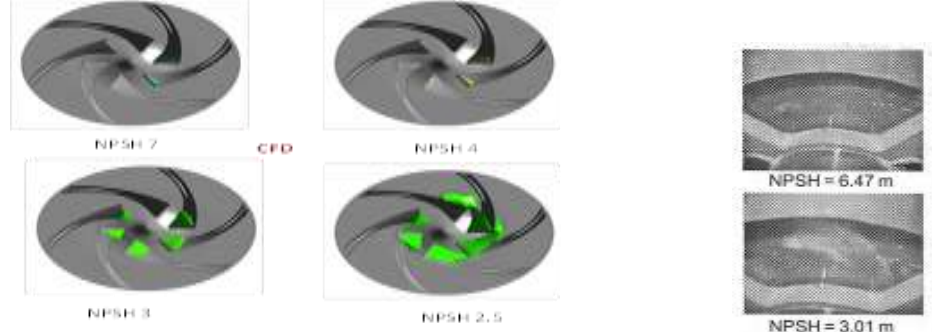

Experiment

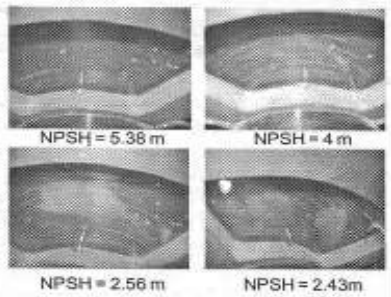

Fig. 10 Comparison of CFD and Experimental [Ref-1] cavitation at 26lps, 1500rpm while decreasing water level

Figure.11 shows cavity length with vapor fringe plot blade to blade view which corresponding to points $\mathrm{A}, \mathrm{B}, \mathrm{C}$ and $\mathrm{E}$ in head drop curve. There is no cavitation when NPSH $=9 \mathrm{~m}$, attach cavitation when NPSH=7 and super cavitation when NPSH $=2.5 \mathrm{~m}$ as shown in figure 11 . Therefore head drop curve in figure 11 can be divided according to cavity length on blade surface into three portions as no cavitation, attach cavitation and super cavitation. No cavitation is range from A to B in figure 11 in this portion rate of vapor production 
approximate zero. Attach cavitation is range from B to C that has cavity length on blade surface less than or equal chord length. Cavitation on suction surface is growing when decreasing NPSH. If cavity length grows up more than chord length, super cavitation occurs at NPSH $2.5 \mathrm{~m}$ as shown in figure 10. Super cavitation is range from $\mathrm{C}$ to $\mathrm{E}$ in figure 11.

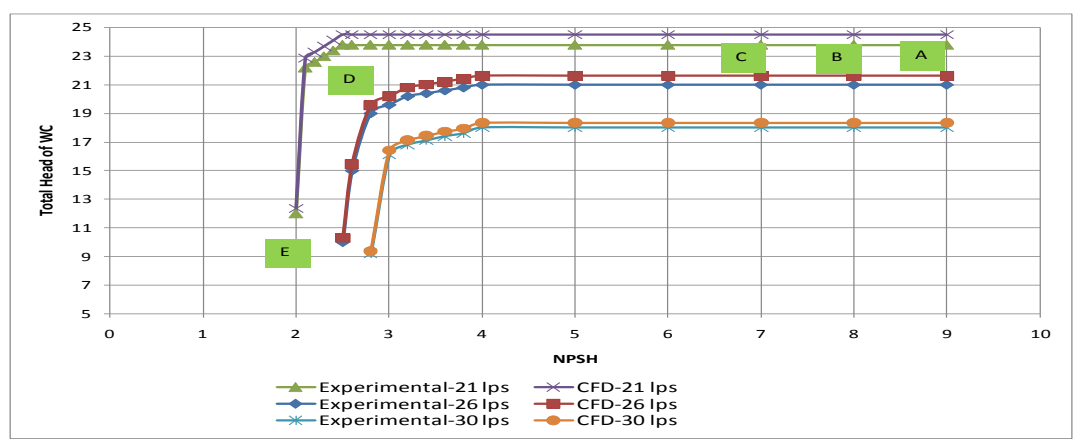

Fig. 11 Comparison of CFD and Experimental [Ref-1] cavitation characteristics for decreasing water level in the cavitation well. Total head V/s NPSH curve at different flow rate at speed $1500 \mathrm{rpm}$

\section{Conclusions}

The flow through the blade passage was numerically studied with CFD code, ANSYS CFX in order to detect formation of cavitation in centrifugal pump and compared with experimental data over the wide flow range. The comparison of the predicted head and experiment data shows a good agreement. Head drop curve has a knee shape that head remain constant while NPSH decreased and head will be rapidly decreased at critical point at point $\mathrm{D}$ (fig 11). The beginning of cavitation in the blade passage can be detected and shown in quality and quantity with numerical simulation.

The inception of cavitation is take place on the suction surface where the leading edges meet the tip. In pressure distribution plot shows that the cavitation zone expanding to the trailing edge especially in super cavitation case (NPSH $2.5 \mathrm{~m}$ ) (fig 10). The available NPSH of the system must be equal to or greater than the NPSH required by the centrifugal pump in order to avoid cavitation difficulties.

\section{References}

[1]. S Christopher and S Kumaraswamy, April 2011 "Experimental Study of Cavitation Hysteresis on Radial Flow Pump" Institution of Engineers (India) Journal-MC, Vol.92, pp.34-39

[2]. Tulin, M. P., (1953), "Steady Two-Dimensional Cavity Flows about Slender Bodies", Tech. Rep., 834, David Taylor Model Basin, Washington, D.C., U.S.A.

[3]. Tulin, M. P., (1955), "Supercavitating flow past foils and struts, Symp, on Cavitation in Hydrodynamics", Teddington, U.K.

[4]. Geurst, J. A., (1960), "Linearized theory for fully cavitated hydrofoils". Int. Shipbuilding Progress, 7, 17-27.

[5]. Uhlman, J. S., (1987), "The surface singularity method applied to partially cavitating hydrofoils", J. Ship Res., Vol. 31, pp. 107124.

[6]. Uhlman, J. S., (1989), "The surface singularity or boundary integral method applied to supercavitating hydrofoils", J. Ship Res., Vol. 33, pp. 16-20

[7]. Kinnas, S. and Fine, N., (1993), "A numerical nonlinear analysis of the flow around two- and three-dimensional partially cavitating hydrofoil", Journal of Fluid Mechanics, 254:151-181.

[8]. Guoyu Wang, Inanc Senocak, Wei Shyy, Toshiaki Ikohagi, Shuliang Cao, "Dynamics of attached turbulent cavitating flows", Elsevier Science Ltd, 2001

[9]. Khin Cho Thin, Mya Mya Khaing, and Khin Maung Aye, "Design and Performance Analysis of Centrifugal Pump", World Academy of Science, Engineering and Technology pp46,2008

[10]. Mohammed Khudhair Abbas, "CAVITATION IN CENTRIFUGAL PUMPS", Diyala Journal of Engineering Sciences in December. 2010

[11]. Shen, Y. T., Dimotakis, P. E., 1989, "The Influence of Surface Cavitation on Hydrodynamic Forces", Proc. $22^{\text {nd }}$ ATTC, St. Johns, pp4-53

[12]. S.Rajendran and Dr.K.Purushothaman, "Analysis of a centrifugal pump impeller using ANSYS-CFX", International Journal of Engineering Research \& Technology (IJERT) Vol. 1 Issue 3, May - 2012

[13]. Ansys CFX solver Modeling guide, Release 13.0 in November 2010

[14]. Bruno Schiavello and Frank C. Visser, "PUMP CAVITATION-VARIOUS NPSHR CRITERIA, NPSHA MARGINS, AND IMPELLER LIFE EXPECT ANCY". Proceeding of the twenty-fifth international pump user Symposium, 2009.

[15]. Larry Bachus., and Angel Custodio. 2003. Known and Understand Centrifugal Pump.Japan: Bachus Company. Tokyo 113

[16]. T. Watanabe, T. Kawamura, Y. Takekosi, M. Maeda, S.H. Rhee, (2003), "Simulation of Steady and Unsteady Cavitation on a Marine Propeller Using a RANS CFD Code", Proceedings of the 5th International Symposium on Cavitation, Osaka, Japan.

[17]. Andrej PREDIN, Cavitation Swirl in the Inlet Pipe of the Radial Pump, Proceedings of the 2nd IASME / WSEAS International Conference on Water Resources, Hydraulics \& Hydrology, Portoroz, Slovenia, May 15-17, 2007

[18]. Park, K., H. Seol, W. Choi and S. Lee, 2009. Numerical prediction of tip vortex cavitation behavior and noise considering nuclei size and distribution". Elsevier Ltd, Applied Acoustics, 70: 674-680.

[19]. Donald, P.S., 2007. Cavitation in High Energy Pumps - Detection and Assessment of Damage Potential, Proceedings of the TwintyThird International Pump Users Symposium, pp: 29-38.

[20]. Al-Arabi, A.B. and S.M.A. Selim, 2007. A Theoretical Model to Predict Cavitation Inception in Centrifugal Pumps, Proceedings of 5th International Conference on Heat Transfer, Fluid Mechanics and Thermodynamics 